\title{
The COVID-19 Pandemic Influencing Prehospital Delay Among Older Adult Patients with Acute Coronary Syndrome Seeking Emergency Care
}

\author{
Audai Hayajneh ${ }^{1}$, Mohammad Rababa ${ }^{1}$, and Sami Al-Rawashdeh ${ }^{2}$ \\ ${ }^{1}$ Jordan University of Science and Technology \\ ${ }^{2}$ The Hashemite University
}

November 22, 2020

\begin{abstract}
Background: The prevalence of prehospital delay is high among older adults with acute coronary syndrome (ACS), resulting in adverse health outcomes. The current study aimed to examine the associated factors of prehospital delay among patients with ACS during the COVID-19 pandemic. Methods: This cross-sectional study was conducted on a convenience sample of 300 older adults with ACS admitted to the emergency department. Bivariate and multivariate analyses, including stepwise linear regression, were used to explore the predictors of prehospital delay and length of stay. Results: Being widowed, education, pain intensity, the gradual onset of ACS symptoms, symptoms lasting for more than 30 minutes, feeling anxious about their ACS symptoms due to the COVID-19 pandemic, perceiving their symptoms as dangerous due to the COVID-19 pandemic, history of myocardial infraction (MI), and mode of transportation were associated with the time taken before seeking emergency care among older adults with ACS during the COVID-19 pandemic. Conclusion: Length of prehospital delay among the participants varied according to certain sociodemographic and clinical characteristics and biomarkers. Improved understanding of the associations between prehospital delay and these characteristics is crucial for achieving optimal ACS patient outcomes under the impacts of the COVID-19 pandemic.
\end{abstract}

\section{Hosted file}

20Nov2020prehospital delay.pdf available at https://authorea.com/users/377802/articles/ 494481-the-covid-19-pandemic-influencing-prehospital-delay-among-older-adult-patientswith-acute-coronary-syndrome-seeking-emergency-care 